\title{
Lectura digital y su influencia en las prácticas lectoras en contextos escolares
}

\author{
Digital Reading and its influence in the Reading practices
}

\author{
Silvia C. Acosta Velázquez ${ }^{a}$ Elba M. Pedraza Amador ${ }^{b}$
}

\begin{abstract}
:
Las Tecnologías de Información y Comunicación (TIC) forman parte cotidiana de los procesos de enseñanza-aprendizaje hoy en día, gracias a su inclusión en el ámbito académico a partir de la segunda mitad del siglo XX; lo que ha permitido que la lectura digital se convierta en una herramienta para el fomento de la lectura y en una práctica que promueve la incorporación de nuevas habilidades y destrezas en el lector.
\end{abstract}

En este artículo se realiza una revisión documental que gira en torno a las aportaciones de la lectura digital desde su inclusión en el ámbito educativo y el cambio que se suscita en el comportamiento y competencias que desarrollan los lectores a partir de su uso.

\section{Keywords:}

Lectura digital, Prácticas lectoras, Competencias, Lectores

\section{Resumen:}

Information and Communication Technologies (ICT) are an everyday part of the teaching-learning processes nowadays, thanks to their inclusion in the academic field since the second half of the 20th century; what has allowed digital reading to become a tool for the promotion of reading and a practice that promotes the incorporation of new skills and skills in the reader.

In this article, a documentary review is made, which revolves around the contributions of digital reading from its inclusion in the educational field and the change that arise in the behavior and skills developed by readers from its use.

\section{Palabras Clave:}

Digital reading, Reading practices, Skills, Readers

\section{Introducción}

La lectura es una actividad que el ser humano inicia desde edades tempranas y se constituye como una herramienta que permite su aprendizaje a lo largo de la vida; sin embargo, su desarrollo no es innato sino que se da principalmente por la inserción en el ámbito educativo y por la interacción de la persona en distintos contextos, lo que da como resultado la adquisición de una amplia gama de actividades que van desde el conocimiento de palabras y gramática, hasta el conocimiento del mundo circundante [1].

Aunado a lo anterior, la lectura es una práctica que no se circunscribe al ámbito educativo sino a la vida cotidiana, cumpliendo una función social y comunicativa, que se da en actividades tan comunes como leer un correo electrónico o el periódico (Cassany, 2006 en Amavizca M., Álvarez-Flores, \& Hernández y Hernández, 2017).

Por su parte, la introducción de las Tecnologías de Información y la Comunicación (TIC) en las actividades diarias de las sociedades ha dado como resultado un cambio en la forma de transmitir y recibir la información, así como en la interacción del ser humano con el ambiente, el cual se ha convertido en un escenario primordialmente visual que se da en medios virtuales, lo que conlleva al individuo a seleccionar, interpretar y analizar información que obtiene de medios digitales [2]. De esta forma se desarrollan capacidades ligadas al uso de la información, las cuales determinan en gran medida

a Autor de Correspondencia, Universidad Autónoma del Estado de Hidalgo, Escuela Superior de Tizayuca, https://orcid.org/0000-00018972-7748, Email: silvia_acosta2066@uaeh.edu.mx

b Universidad Autónoma del Estado de Hidalgo, Escuela Superior de Tizayuca, https://orcid.org/0000-0002-7182-2437 , Email: elbam@uaeh.edu.mx 
el acceso al conocimiento, por lo que este texto se centra en la influencia de la lectura digital en las prácticas lectoras.

\section{Evolución del texto impreso al texto digital}

A lo largo de la historia de la humanidad, la lectura ha estado presente en diferentes formas, principalmente como una práctica colectiva llevada a cabo en voz alta, que sufrió una profunda transformación a partir de la invención y difusión de la imprenta a mediados del siglo $\mathrm{XV}$, momento en el que la producción de libros dio lugar a que un mayor número de personas tuviera acceso a textos impresos. Lo anterior permitió que el texto impreso fuera reconocido como el principal medio de transmisión de información, lo que generó cambios sustanciales en los hábitos de lectura al convertirse en una práctica individual. En el siglo XVIII la lectura era un privilegio que gozaba sólo una parte de la población, que contaba con capacidad para acceder a la compra de libro, en tanto que para el resto se convertía en una actividad social que tenía lugar en los sitios de trabajo; sin embargo, también fue el siglo en que se empezó a industrializar y a comercializar [3].

Esta posición se mantuvo hasta que el fenómeno de la digitalización, con la introducción de las computadoras y posteriormente del Internet, provocara la transición del papel al mundo virtual y con ello al surgimiento de una nueva forma de leer [4].

Es así que, el Internet creo un nuevo espacio para acceder al conocimiento más allá de las fronteras geográficas y esto transformó medios como los periódicos y otras publicaciones que pasaron del papel a ser digitales [5].

El libro electrónico surge en la década de los 70's con la aparición del Ilamado Proyecto Gutenberg, cuyo objetivo era hacer llegar a más personas las obras literarias y fue tal su potencial que a finales de la década de los 90's, su creador Michael Hart ya visualizaba el potencial del texto electrónico como el nuevo medio de comunicación [6] pues con él surgen oportunidades de negocio en el ambiente digital, las cuales fueron aprovechadas por empresas como Amazon.com, quien toma el sistema de asociados para la venta en línea de libros.

La digitalización favoreció la rapidez en el proceso de producción, lo que ha reunido a otros actores articulados al proceso tales como editores, diseñadores gráficos, prensa, entre otros, además de permitir el acceso a información en diferentes idiomas, extendiéndose a muy diversos ámbitos, entre ellos el académico.

\section{EI acceso a las TIC y su relación con los libros digitales}

La lectura en medios electrónicos es una práctica cada vez más frecuente en la sociedad actual. Si bien es cierto que su utilización requiere el desarrollo de competencias adicionales, relacionadas con el manejo de herramientas digitales, ofrece también la oportunidad de potenciar y desarrollar las habilidades de comprensión lectora en un ambiente que promueve la interacción, la comunicación y el aprendizaje.

Sin embargo, los medios digitales para lectura aun con el auge que han tenido, no sustituyen de ninguna manera a los textos impresos, sino más bien los auxilian y complementan, refieren nuevas formas de enseñar y de aprender y ofrecen mayores opciones a los lectores, cuyo interés se despierta a partir de la combinación de los diferentes estímulos (sonidos, colores, movimiento) y la interacción con el texto a que obliga la navegación en internet.

La disponibilidad de tecnología y el acceso de la población a ella ha sido determinante en la masificación de la lectura y por ende en los cambios en el consumo de contenidos y en la adopción de dispositivos para la lectura de textos electrónicos. Tan sólo en México, de acuerdo con datos sobre hábitos de los usuarios de internet en México [7] se observa que en el año 2007 había cerca de 23.9 millones de internautas en el país y para el año 2017 se incrementó hasta 79.1 millones lo que representa más de tres veces el crecimiento y con ello se ha llegado a un $67 \%$ de penetración entre la población mayor de 6 años. Dentro de ese grupo el 54\% de la población se encuentra en un rango de edad entre los 12 y los 34 años.

De igual forma el estudio muestra que la preferencia de los internautas en cuanto a dispositivos de conexión a Internet es el smartphone (76\%) seguido por las laptops (49\%), siendo el acceso a las redes sociales la principal actividad realizada por un $89 \%$ de los internautas, sin embargo, se destaca que el $82 \%$ busca información y $65 \%$ lee, ve o escucha contenido relevante, lo que implica actividades relacionadas con la lectura en medios digitales.

No obstante, los avances que se han tenido en cuanto al acceso de los mexicanos a las TIC, siguen existiendo brechas tales como las condiciones para descarga de contenidos digitales lo que dificulta que los 
usuarios puedan aprovechar el cúmulo de información que hay en la Red. El mismo estudio señala que $50 \%$ de los internautas en México considera que la conexión es lenta o que hay poca oferta de proveedores de servicio de Internet, mientras que el $38 \%$ considera que el costo es elevado.

Pese a estos obstáculos los lectores muestran más interés en usar medios digitales para acercarse a la lectura. Por su parte, la Primera Encuesta Nacional Sobre Consumo Digital y Lectura realizada en México en el año 2015, que tenía el objetivo de conocer el impacto de los medios digitales en la cultura lectora y los intereses de los jóvenes en la lectura en medios digitales e impresos, refiere que la mayor parte de los encuestados, jóvenes entre 12 y 29 años, cuentan con un Smartphone para su uso personal (97\%) y permanecen conectados a internet durante 6 horas aproximadamente al día, refiriéndose un tiempo mayor en estudiantes de educación superior [8, p. 22].

Cabe destacar que los resultados de la encuesta apuntan a que "internet también es una herramienta de acceso a la lectura por iniciativa" pues el $68 \%$ de los estudiantes universitarios encuestados lo usan para investigar temas de interés y el $60 \%$ para leer noticias, usando también este medio para la lectura de libros y revistas en línea [8, pp. 46-47].

Lo anterior concuerda con un estudio realizado por la UNESCO (2015) sobre la lectura móvil en los países en desarrollo, en el cual participaron siete países, destacándose Ghana, Etiopía y Pakistán. Dicho estudio refiere que los usuarios leen en sus teléfonos móviles principalmente por comodidad al traerlo consigo, lo cual garantiza el acceso al contenido, facilita la portabilidad y asequibilidad en comparación con los libros impresos, considerándose entonces que, para un lector avanzado, los libros electrónicos representan un ahorro.

Aunado a lo anterior el mismo informe [9, pp. 39-50] muestra que hay cambios positivos en las personas que ya gustaban de la lectura, pues con el uso de los libros electrónicos en teléfonos celulares reforzaron el gusto; así mismo, en el caso de las personas a quienes les disgustaba, se encontró una tendencia al cambio de actitud, ya que quienes lo hacían por primera vez experimentaron una mejor experiencia, además que quienes ya habían adoptado la lectura digital reportaron un cambio en la frecuencia de lectura por la accesibilidad que representan los textos digitales a través de teléfonos móviles. Esto indica que los medios digitales se muestran como una alternativa para el fomento a la lectura.
Adicionalmente a lo expuesto con anterioridad, surge la alfabetización como una prioridad, en tanto que permite la construcción de significados [10], el tránsito de poder leer a saber leer y escribir, de decodificar a comprender y ser capaz de producir textos, más aún en el siglo XXI que con la rapidez de los cambios en la tecnología no sólo se requiere leer, sino escribir, comprender y manejar los dispositivos tecnológicos que son soporte de lectura [11].

Al r3specto, las estadísticas señalan que en México para el 2015 [12] el 5.5\% de la población de 15 años y más es analfabeta, siendo las mujeres el grupo de población más afectado; 6 de cada 100 mujeres no saben leer ni escribir, lo cual constituye un reto para el sistema educativo, cuya alternativa de apoyo puede estar en el uso de dispositivos móviles y libros digitales.

\section{El libro digital en el contexto escolar}

En el medio escolar, la coexistencia de las prácticas de lectura en libros y materiales impresos ha suscitado controversias entre docentes y alumnos debido a que tradicionalmente, el objetivo de las escuelas es transmitir conocimientos a los estudiantes a través de la lectura mediada por un emisor (docente), que acostumbrado al libro de texto impreso como recurso principal, desarrolla resistencia y frustración al adoptar el papel de facilitador y convertirse en una mera herramienta del proceso de enseñanza-aprendizaje en un escenario digitalizado [13, pp. 2-3], además del esfuerzo adicional que le supone la planificación y organización de los contenidos escolares $y$ los conocimientos requeridos sobre recursos tecnológicos para abordar la lectura apoyada en soportes digitales.

Por otro lado y en cuanto a la experiencia de leer se refiere, los estudiantes, nativos digitales en su mayoría, transforman el proceso de lectura con la utilización de dispositivos que si bien les resultan conocidos, no han aprendido a usar de manera sistemática, sino más bien de forma autónoma, con lo que se incrementa el riesgo de que utilicen indiscriminadamente la tecnología para fines diferentes a actividades académicas, redundando en un uso incorrecto, inadecuado y poco eficaz de ella [13, p. 4].

De acuerdo con Viñao, A. (2010), lo digital se puede percibir como un espacio donde aparecen nuevos modos de leer la realidad, por lo que aun cuando la utilización de soportes tecnológicos para realizar la lectura no asegura un incremento en la calidad de los aprendizajes, se manifiestan sus ventajas en el ámbito académico, al constituirse como una herramienta que favorece la 
mejora del aprendizaje y contribuye a la generación de determinados procesos mentales en las personas.

En lo que respecta a las tecnologías que de manera intencional se crean para ser utilizadas en el contexto educativo, éstas brindan apoyo a los procesos cognitivos y auxilian en la generación del interés de los alumnos por la lectura. De igual forma contribuyen a desarrollar en ellos la creatividad y el espíritu de investigación, además de favorecer al desarrollo de su capacidad de análisis y síntesis, dando lugar a una transformación en las formas de comunicación y proceso de información.

En este sentido, se señala que los libros digitales diseñados para el ámbito educativo "responden a una visión estructurada del conocimiento, similar a los libros de texto impresos, pero incorporando la interactividad, el lenguaje audiovisual e iconográfico y la hipertextualidad" [14, p. 232], con la finalidad de hacer atractiva la información para los estudiantes, que además de lectores, adoptan un rol de usuarios, receptores activos y constructores de su propio conocimiento.

Pese a las ventajas que ofrece el libro digital, también llamado libro electrónico, el usuario debe realizar actividades diferentes a las que le ofrece un libro impreso, ya que un formato de texto que incluye hipervínculos, video u otras funciones demanda del lector un procesamiento más dinámico de la información [15]. Además otras investigaciones [16] refieren cambios en los hábitos de lectura en estudiantes, quienes son más propensos a leer documentos impresos de una sola vez y a profundidad, en tanto que al realizar una lectura digital usan atajos de navegación por palabras clave y selectividad, lo cual puede llevar a una deficiente comprensión y limitar el progreso académico.

El desafío para los estudiantes es aprender a leer de manera comprensiva y reflexiva, tanto de forma tradicional, haciendo uso de libros de texto y materiales impresos como de manera digital, para acceder al conocimiento en cualquier disciplina. Complementariamente, si el estudiante desarrolla el interés por la lectura, lo hará con los medios que tenga a su alcance, sean éstos impresos o digitales, lo cual es una muestra de que no se discriminan las aportaciones del modelo tradicional de lectura, sino que con el uso de los medios digitales y la tecnología disponible, surgen nuevas formas de leer que posibilitan que los contenidos de los textos "lleguen" a un número cada vez mayor de lectores y que éstos puedan incluso interactuar entre sí o acceder de manera simultánea a un mismo texto sin necesidad de acudir a una biblioteca para realizar la actividad de lectura.
De igual forma, la inclusión de las TIC, entre ellas los libros digitales y otras herramientas demandan el replanteamiento de modelos, planes y prácticas educativas con la finalidad de no ser sólo un accesorio más, sino de que sean realmente provechosos para el proceso de enseñanza-aprendizaje.

Sin embargo, a pesar de lo expuesto surge un cuestionamiento en relación a la efectividad de los medios digitales para lograr la comprensión de lo que se lee, mismo que será abordado en el siguiente apartado.

\section{Cambios en las prácticas lectoras}

Como ya mencionó anteriormente, hoy en día no es suficiente saber leer sino comprender y utilizar adecuadamente los recursos digitales y dispositivos electrónicos para localizar y seleccionar la información requerida para el desarrollo de las actividades [17], y en el ámbito escolar se refiere que es preciso asegurar que los estudiantes aprendan el funcionamiento de las nuevas tecnologías para mejorar sus aprendizajes [18, p. 5]; sin embargo, uno de los objetivos que se persiguen en los entornos digitales es la adquisición de conocimiento desde una variedad de fuentes, lo que implica el desarrollo de habilidades para lograr una comprensión del texto digital.

Al respecto, Salmerón, Strømsø, [19] proponen el desarrollo de competencias que permitirán la comprensión en la lectura digital (Figura 1).

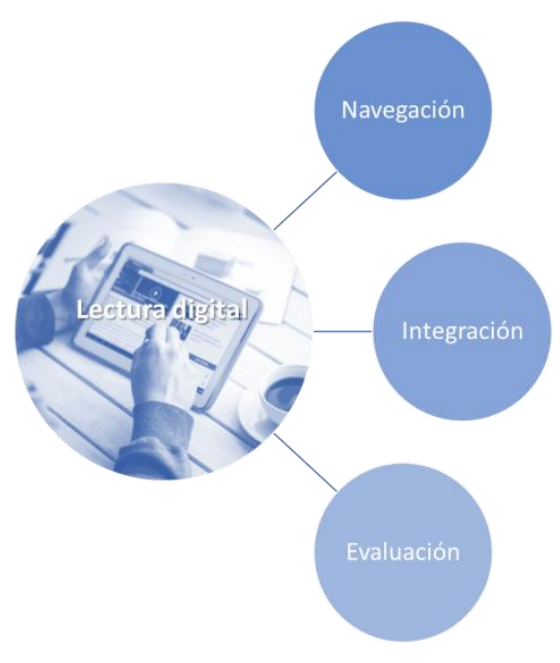

Figura 1. Competencias para la comprensión de la lectura digital.

Fuente: Elaboración propia con información de [19]

La navegación involucra la búsqueda y escaneo de información relevante y el acceso a motores de 
búsqueda para crear consultas, lo que implica un cambio en el comportamiento de los usuarios, que son motivados en cierta medida por el diseño de navegación del sitio o por el formato de texto.

La integración se refiere a la capacidad de los lectores para construir una representación mental basada en la asociación del conocimiento previo con la nueva información. Esto requiere la identificación y selección de la información, así como procesos de inferencia por parte del lector. Leer en medios digitales implica que los lectores hagan un uso eficiente de su capacidad cognitiva y de su habilidad para realizar múltiples tareas.

La evaluación está relacionada con la capacidad de determinación de la confiabilidad de los contenidos y fuentes, ya que los lectores toman decisiones mediante la comparación de lo que leen con sus creencias y conocimientos previos.

Como se observa, debe considerarse también un análisis relativo a los procesos cognitivos implicados en la comprensión lectora, basados principalmente en la percepción, la atención y la memorización, necesarios para la interpretación de textos, y de manera relevante la concentración, que es una capacidad que, en la actualidad, debido al excesivo uso de tecnología, los alumnos no desarrollan fácilmente [13].

Por otra parte, y debido a que la participación en la sociedad actual involucra de manera permanente actividades de lectura y manejo de información en ambientes virtuales, con el propósito de estar a la vanguardia se plantea también una ruptura con los tradicionalismos eistentes en los procesos formativos, ya sea en el ámbito escolar o laboral, pues debe reconocerse que la inclusión de textos digitales en los diferentes escenarios implica una redefinición de los procesos mentales requeridos para su proceso e interpretación.

De igual manera, la incorporación de medios digitales en el proceso de enseñanza aprendizaje constituye un reto para el profesorado, que requiere apoyarse de procesos de capacitación y adopción de las TIC. Considérese que los docentes que han accedido a dicha incorporación, se ven más motivados a conectar con los estudiantes que son nativos digitales [20]. Además de considerar que la enseñanza con libros digitales implica una planeación que inicia con la necesidad de asociar los medios digitales al contexto del aula.

\section{Conclusiones}

Tras el análisis realizado, se asume que los avances tecnológicos han impactado a los procesos de lectura y plantean incluso una ruptura con los paradigmas tradicionales.

Si bien es cierto que a partir de la invención de la imprenta, la producción en serie de libros marcó una transformación en la sociedad al dar apertura a que los no lectores accedieran a la información y al conocimiento, las actuales tendencias en la economía, los procesos globalizadores y de internacionalización de los mercados marcan nuevas pautas y representan desafíos cada vez más elevados para la sociedad contemporánea.

Considérese aquí que la lectura hoy en día se encuentra implicada no sólo en las actividades académicas, sino también en aquellas realizadas en otros contextos y constituye el acceso a la información y al conocimiento, por tanto, se requiere no sólo saber pasar la vista por las líneas, sino lograr la comprensión de lo que se lee, por tanto, resulta indispensable replantear las prácticas lectoras, dejando en claro la necesidad de fomentar el desarrollo de nuevas competencias y saberes ligados al uso de la información.

Desafortunadamente, las modificaciones en la educación privilegian el uso de tecnología en las aulas para auxiliar los procesos de enseñanza-aprendizaje sin tomar en cuenta una formación básica en su uso y manejo, que en la mayoría de los casos se fundamenta en la experiencia o el aprendizaje autónomo, desprovisto de parámetros que conduzcan a una utilización adecuada y eficiente.

Adicionalmente, a pesar de los avances tecnológicos, no existe una cultura de fomento a la lectura ni se considera, dentro de los programas educativos, la enseñanza de la lectura múltiple, es decir, enseñar a las personas a leer bajo modelos tradicionales apoyados en textos impresos y en ambientes digitales, mediados por dispositivos electrónicos. Esto representa un grave desafío y una barrera para el desarrollo humano, que se ha hecho aún más evidente en las últimas dos décadas.

No se trata pues de privilegiar la lectura en ambientes virtuales en detrimento de la lectura tradicional, pues aún con el auge de los textos digitales, es un hecho que los textos impresos siguen siendo reconocidos como el medio para transmisión de información y acceso al conocimiento por excelencia, sino asumir que es una acción complementaria dentro de un escenario donde coexisten dos modelos igualmente importantes que 
deben ser tratados como tales, para logar una contribución real al desarrollo humano y de la sociedad.

\section{Referencias}

[1] OCDE, Marco de Evaluación y de Análisis de PISA para el Desarrollo: Lectura, Matemáticas y Ciencias, París: OECD Publishing, 2017.

[2] N. Escobar, E. Manyoma y Y. Gómez, «La lectura digital como estrategia para el fomento de la producción de textos académicos,» de Memorias de la Décima Quinta Conferencia Iberoamericana en Sistemas, Cibernética e Informática (CISCI), 2016.

[3] B. W. Cull, «Reading revolutions: Online digital text and implications for reading in academe.,» First Monday, 2011.

[4] A. Gutiérrez V., «E-reading, la nueva revolución de la lectura: del texto impreso al ciber-texto,» Revista Digital Universitaria, vol. 7, no 5 , pp. 1-8, 2006.

[5] M. Lebert, A short history of eBooks, Toronto: University of Toronto, 2009.

[6] M. Lebert, Project Gutenberg's Del libro impreso al libro digital, Toronto: NEF, Universidad de Toronto, 2010.

[7] Asociación de Internet, «14 Estudio sobre los Hábitos de los usuarios de Internet en México 2018,» México, 2018.

[8] IBBY, «Primera Encuesta Nacional Sobre Consumo Digital y Lectura,» Noviembre 2015. [En línea]. Available: www.loquesea.com. [Último acceso: 24 Junio 2016].

[9] UNESCO, «La lectura en la era móvil: Un estudio sobre la lectura móvil en los países en desarrollo,» 2015. [En línea]. [Último acceso: 28 Mayo 2016].

[10] C. Amiama-Espaillat y C. Mayor-Ruiz, «Lectura digital en la competencia lectora:,» Comunicar. Revista Científica de Comunicación, vol. XXV, nº 52, pp. 105-114, 2017.

[11] UNESCO, «La lectura en la era digital: Un estudio sobre la lectura móvil,» México, 2015.

[12] INEGI, «Cuéntame...población,» 16 enero 2019. [En línea]. Available: http://cuentame.inegi.org.mx/poblacion/analfabeta.aspx?tema=P.

[13] M. Palomar, Resistencias e integración de las TIC en los Centros Educativos de Secundaria. Innovación y Experiencias Educativas, 2010.

[14] M. M. Area y M. Á. Marzal, «Entre libros y pantallas. Las bibliotecas escolares ante el diseño digital,» Revista de Currículum y Formación de Profesorado [en linea], enero - abril 2016.
C. López-Andrada, «Entre la reproducción y la obsolescencia: investigaciones y prácticas con libros electrónicos y dispositivos de lectura en entornos educativos,» PAAKAT: Revista de Tecnología y Sociedad, n 12, 2017.

[16] M. Wolf, «Our 'Deep Reading' Brain: Its Digital Evolution Poses Questions,» Nieman Foundation for Journalism at Harvard University, 2010.

[17] A. Vilches y C. Furió, «Ciencia, Tecnología, Sociedad: Implicaciones en la Educación Científica para el siglo XXI,» Campus OEI, 15 marzo 2016.

[18] P. Marques, El papel de las TIC en el proceso de lecto-escritura Leer y escribir en la escuela... a golpe de clic, Planeta, 2006.

[19] L. Salmerón, H. I. Strømsø, Y. Kammerer, M. Stadtler y P. van den Broek, «Comprehension processes in digital reading,» de Learning to read in a digital world, Amsterdam, John Benjamins, 2018, pp. 91-120.

[20] S. Amavizca M., E. P. Álvarez-Flores y D. Hernández y Hernández, «Lectura digital en la universidad: entre lo cotidiano y lo académico,» Estudios Lambda. Teoría y práctica de la didáctica en lengua y literatura, pp. 46-71, 2017

[21] A. Viñao, «Memoria, patrimonio y educación,» Educatio Siglo XXI, vol. $28, \mathrm{n}^{\circ} 2$, pp. $17-42,2010$

[22] P. C. Cerrillo-Torremocha, «Nuevos tiempos, ¿nuevos lectores?,» Revista OCNOS, pp. 19-33, 2005.

[23] I. P. L. I. García y L. S. R. L. A. y. H. K. Jhonson, «Informe Horizon, Edición Iberoamericana,» The New Media Consortium, Austin, Texas, 2010.

[24] AMIPCI, «11o. Estudio sobre los hábitos de los usuarios de internet en México 2015,» 2015. [En línea]. [Último acceso: 19 Junio 2016]. 